\title{
Diagnóstico clinico e subsidiário da incontinência urinária
}

\author{
Clinical and subsidiary diagnosis of urinary incontinence
}

Paulo Cezar Feldner $\mathrm{Jr}^{1}$, Marair Gracio Ferreira Sartori² ${ }^{2}$ Geraldo Rodrigues de Lima $^{3}$, Edmund Chada Baracat ${ }^{3}$, Manoel João Batista Castello Girão ${ }^{4}$

\section{RESUMO}

Os sintomas do trato urinário feminino são inespecíficos e se faz necessário correta investigação clínica para se estabelecer o diagnóstico da incontinência urinária. Tal avaliação consiste em história clínica, questionário de qualidade de vida, exame físico, diário miccional, teste do absorvente e estudo urodinâmico. O estudo urodinâmico é extensão da história e do exame físico para melhor avaliar a etiologia das queixas das pacientes. O objetivo deste artigo é revisar os métodos utilizados para o diagnóstico clínico e subsidiário da incontinência urinária.

PALAVRAS-CHAVE: Incontinência urinária; Diagnóstico clínico; Urodinâmica

\section{ABSTRACT}

Female lower urinary tract symptoms are nonspecific and a clinical evaluation is required to establish the correct diagnosis. Such evaluation should consist of a structured micturition history or questionnaire, physical examination, micturition diary, pad test, and urodynamic evaluation. Urodynamic investigation was developed as an extension of patient history and physical examination in order to reveal the etiology of the patient's complaints. The goal of the present article is to review clinical and subsidiary diagnosis of urinary incontinence.

KEYWORDS: Urinary incontinence; Diagnosis, clinical; Urodynamics

\section{Introdução}

Recentemente, a Sociedade Internacional de Continência (ICS) publicou novas definições dos sintomas, sinais, observações urodinâmicas e condições associadas com as disfunções do trato urinário baixo e do estudo urodinâmico. Estas definições foram adotadas para serem compativeis com as publicações da Organização Mundial de Saúde e a Classificação Internacional de Doenças (CID-10), promovendo melhor comunicação e entendimento entre clínicos, pesquisadores e o público em geral ${ }^{1}$. Desta forma podemos ter: incontinência urinária (IU), como a queixa de perda involuntária de urina; incontinência urinária de esforço (IUE), como a queixa de perda involuntária de urina sincrônica ao esforço, espirro ou tosse; incontinência de urgência, como a queixa de perda involuntária de urina associada ou imediatamente precedida por urgência miccional; incontinência urinária mista, como a queixa de perda involuntária de urina associada com urgência e também com o esforço, espirro ou tosse.

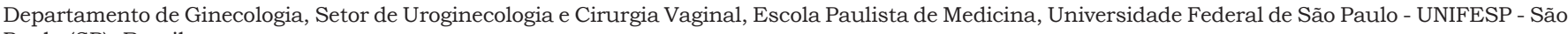
Paulo (SP), Brasil.

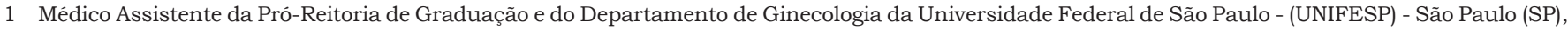
Brasil.

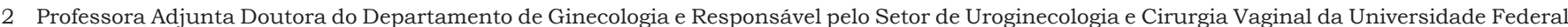
de São Paulo - (UNIFESP) - São Paulo (SP), Brasil.

3 Professor Titular do Departamento de Ginecologia da Universidade Federal de São Paulo - (UNIFESP) - São Paulo (SP), Brasil.

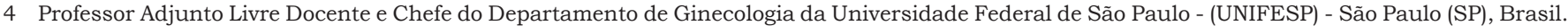

Correspondência: Paulo Cezar Feldner Jr

Rua Mirassol, n 272, apto 61 - Vila Clementino - 04.044-000 - São Paulo - SP - Telefone/Fax: (11) 5084-2514 - e-mail: pfeldner@alfa.epm.br 
A bexiga hiperativa é classificada como sindrome de sintomas sugestivos de disfunção do trato urinário baixo. É, especificamente, definida como urgência, com ou sem urgeincontinência, usualmente acompanhada de aumento da freqüência miccional e noctúria.

Nesta nova padronização, termos como síndrome da bexiga hiperativa, sindrome de urgência ou sindrome de urgência-freqüência denotam a mesma enfermidade. De acordo com a nomenclatura, estes sintomas combinados são sugestivos de hiperatividade do detrusor (definido por meio de exame urodinâmico como contrações vesicais involuntárias demonstráveis). Ainda seguindo as recomendações da padronização, estes termos só podem ser usados na ausência de infecções ou de outras enfermidades locais.

\section{História Clínica}

A anamnese deve conter alguns aspectos incluindo o início dos sintomas, duração, gravidade, condições associadas e descrição do impacto na qualidade de vida da mulher. Os sintomas vão refletir alteração na fisiologia normal do trato urinário ${ }^{2}$.

Embora a história forneça diversas informações, é freqüente que não se obtenha o diagnóstico, uma vez que os sintomas urinários podem ser similares quando as etiologias são diferentes. Isto faz a história clínica, embora integrante da avaliação uroginecológica, constituir-se apenas de segmento da avaliação total da paciente ${ }^{3}$.

Estudos pioneiros observaram que pacientes com sintoma isolado de perda aos esforços, na ausência de outro sintoma urinário, usualmente têm bexigas estáveis. Demonstraram também alta acurácia da história no diagnóstico de IUE e concluíram que o estudo urodinâmico não é obrigatório nestas situações ${ }^{4}$. Contudo, outros pesquisadores relataram fraca correlação entre os sintomas e os achados urodinâmicos. Estes últimos estudos preconizam o exame urodinâmico na identificação das causas de incontinência ${ }^{5-8}$. Segundo alguns autores, uma em nove mulheres pode ser operada desnecessariamente quando o diagnóstico baseia-se apenas em dados de clínicos ${ }^{9}$.

A história clínica apresenta valor preditivo baixo em relação ao diagnóstico urodinâmico final, em revisão sistemática da literatura ${ }^{7,10}$. Os autores concluíram que pacientes com queixa de perda urinária ao esforço, na avaliação urodinâmica, podem ter o componente de esforço, contrações não inibidas ou mesmo a coexistência de ambos. Assim, a queixa de perda urinária ao esforço tem sensibilidade de 0,82 e baixa especificidade $(0,57)$, resultando em elevados índices de falso-positivo. Baseada nestes dados, a confiança somente em dados da história clínica pode levar a até $25 \%$ de erro diagnóstico. Deve-se lembrar, também, que aumentos na pressão abdominal podem desencadear contrações não inibidas do detrusor, falseando o diagnóstico.

Embora alguns autores tenham encontrado bexigas instáveis em apenas $3,6 \%$ das pacientes com o sintoma puro de perda ao esforço, sugerindo não haver necessidade de complementação com exame urodinâmico ${ }^{4}$, outros pesquisadores observaram que este sintoma puro como queixa única é muito incomum, estando presente em apenas $2 \%$ dos $\operatorname{casos}^{11}$. Em série de casos de pacientes atendidas no setor de Uroginecologia da UNIFESP/EPM, os sintomas mistos foram os mais prevalentes $(52,6 \%)$ e não houve pacientes com hiperatividade do detrusor entre aquelas com sintoma único de perda aos esforços. Da mesma forma, aquelas com queixa de urgência/urgeincontinência isolada não apresentavam IUE na avaliação urodinâmica ${ }^{12}$.

Para o diagnóstico diante da suspeita de bexiga hiperativa, é importante, durante a anamnese, pesquisar o tipo de perda de urina, fatores que pioram ou desencadeiam a perda, tempo de sintomatologia, tratamentos prévios, doenças associadas e medicamentos em uso ${ }^{2}$.

Queixas neurológicas como diminuição da perda muscular, dificuldade na marcha, parestesia de membros inferiores e dificuldade para esvaziar a bexiga devem ser valorizados, uma vez que doenças como esclerose múltipla e doença de Parkinson podem manifestar-se pela primeira vez como queixas urinárias. Antecedentes de traumas e cirurgias raquimedulares também são importantes.

Os sintomas mais comuns entre os casos com bexiga hiperativa são urgência e aumento da freqüência miccional, encontrados em cerca de $80 \%$ das pacientes. Outros sintomas relatados são urgeincontinência e perda urinária durante relação sexual, particularmente no orgasmo ${ }^{13}$.

Para a predição de hiperatividade vesical, a história clínica tem niveis de sensibilidade de 0,69 e de especificidade de $0,60^{10}$. De acordo com a nova padronização da terminologia da ICS, isto implica que, na prática clínica, a bexiga hiperativa é diagnóstico obtido pela clínica e que pode ser usado como base para o manejo inicial, após avaliação dos sintomas individuais do trato urinário baixo, achados de exame físico, exame de urina e outras investigações necessárias ${ }^{1}$.

Para a incontinência mista, a baixa sensibilidade $(0,51)$ e especificidade $(0,66)$ da história 
clínica geram elevados índices de falso-positivo e falso-negativo, com valores preditivos de acerto em apenas $25 \%$ dos $\operatorname{casos}^{10}$. Há que se lembrar, também, que a quantificação clínica da perda urinária é subjetiva, já que ela se baseia nas informações prestadas pela paciente e o impacto dessa perda na qualidade de vida é muito variável, conforme dados culturais, socioeconômicos e raciais. A Tabela 1 evidencia os valores de sensibilidade, especificidade, valor preditivo positivo e negativo dos dados de história clínica no diagnóstico da incontinência urinária.

Tabela 1 - Sensibilidade, especificidade e valores preditivos positivo e negativo da história clínica para o diagnóstico da incontinência urinária (adaptado de Jensen et al. ${ }^{7}$ e Colli et al. ${ }^{10}$ ).

\begin{tabular}{ccccc}
\hline & Sensibilidade & Especificidade & VPP & VPN \\
\hline IUE & 0,82 & 0,57 & 0,74 & 0,77
\end{tabular}

Hiperatividade

\begin{tabular}{|c|c|}
\hline Vesical & 0,69 \\
\hline
\end{tabular}

IUM $\quad 0,51 \quad 0,66 \quad 0,24 \quad 0,84$

IUE: Incontinência urinária de esforço.

IUM: Incontinência urinária mista.

VPP: Valor preditivo positivo.

VPN: Valor preditivo negativo.

O diário miccional é outra ferramenta importante quando se suspeita de hiperatividade vesical. Além de auxiliar no correto diagnóstico, permite a avaliação da gravidade dos sintomas, além de constituir recurso para avaliar terapêuticas utilizadas. É fundamental que se tenha o registro dos medicamentos em uso pela paciente, uma vez que determinados fármacos podem piorar ou mesmo desencadear os sintomas ${ }^{2}$.

Durante o preenchimento do diário, a paciente deve ser orientada a anotar a freqüência miccional (diurna e noturna), volume líquido ingerido, volume urinado, número de episódios de perda urinária, enurese noturna, urgência miccional ou perda durante relação sexual, bem como a quantidade de absorventes utilizados diariamente. A duração do diário miccional pode ser de 3, 5, 7 ou 14 dias, de acordo com a maioria dos autores. Existem divergências sobre o real valor deste tipo de registro, bem como da duração ideal ou mesmo do valor discriminatório e da reprodutibilidade das informações obtidas. Contudo, os diários miccionais ainda continuam sendo largamente utilizados para o diagnóstico e manejo da incontinência urinária ${ }^{14,15}$.

\section{Exame Físico}

O exame físico faz parte da rotina de investigação ginecológica da mulher incontinente, vi- sando reproduzir e caracterizar a incontinência, excluir distúrbios neurológicos, avaliar o suporte pélvico e excluir outras enfermidades pélvicas ${ }^{2}$.

Durante o exame, alguns aspectos devem ser ressaltados: a análise da mucosa vaginal; sinais de dermatite amoniacal; sinais de atrofia pelo hipoestrogenismo; força de contração voluntária dos músculos do assoalho pélvico; tônus do esfincter retal; sensibilidade do períneo e reflexos sacrais, além de eventuais distopias genitais.

A IUE está comumente associada ao prolapso de órgãos pélvicos, sendo importante sua avaliação. Porém, deve-se ter em mente que a presença ou ausência de prolapso genital nada revela sobre a causa da incontinência. Relevante procidência da parede vaginal anterior, por exemplo, pode existir independente ou concomitantemente com a IUE, hiperatividade do detrusor ou ambos.

Alguns autores têm proposto que a história associada à demonstração clínica da perda urinária ao esforço é suficiente para o diagnóstico de $\mathrm{IUE}^{16}$. Esta visão vem sendo modificada pela grande maioria dos pesquisadores em estudos investigando a relação entre este sinal clínico e o diagnóstico urodinâmico final ${ }^{12,17,18}$. A acurácia do sinal clínico de perda ao esforço foi comparada ao estudo urodinâmico em 863 pacientes. Os autores concluíram que o mesmo está presente em apenas $54,2 \%$ das 574 mulheres com diagnóstico final de IUE. Além disso, 38\% tinham outro diagnóstico adicional, como hiperatividade vesical. Observaram que o sinal clínico tem valor preditivo positivo de $91 \%$ e valor preditivo negativo de $50 \%{ }^{17}$.

Mesmo com elevado valor preditivo positivo, em nenhum dos trabalhos há elementos que indiquem a possibilidade de diferenciação da IUE por defeito esfincteriano, o que seria importante na escolha do tratamento cirúrgico.

Em contraposição, outros pesquisadores demonstraram que o sinal clínico tem valor preditivo positivo de $68,2 \%$ e valor preditivo negativo de $88,6 \%$, indicando que a ausência de perda urinária ao exame clínico é mais útil na exclusão do diagnóstico que a sua presença na confirmação ${ }^{18}$.

Em estudo no qual se correlacionou o valor da queixa clínica com o exame físico no diagnóstico da incontinência urinária, observou-se que o sinal clínico de perda urinária ao esforço estava presente em $43,8 \%$ do total de mulheres, sendo que $82 \%$ tinham o componente de esforço ao estudo urodinâmico. O mesmo, no entanto, estava ausente em $56,2 \%$ dos casos, sendo que o componente de esforço foi observado em $46,9 \%$ destas pacientes. Os valores preditivos positivo e negativo do sinal clínico para o diagnóstico de IUE, neste grupo, foram de 82 e 53,1\%, respectivamente ${ }^{12}$. 
Conclui-se, portanto, que a literatura não é unânime na utilização do sinal clínico da perda urinária como único método para diagnóstico da incontinência urinária.

A presença de hiperatividade do detrusor associada à IUE é importante fator de risco na predição do sucesso terapêutico e deve ser avaliada a possibilidade de tratamento clínico antes de cirurgia. Alterações na micção, como a hipocontratilidade do detrusor ou mesmo obstruções infravesicais, também são importantes achados, uma vez que estas mulheres têm risco aumentado para retenção urinária no pós-operatório.

Em ampla revisão sobre o tema, conclui-se que a avaliação urodinâmica deve ser realizada especialmente quando a cirurgia é opção ao tratamento. Segundo os autores, apenas quando a terapia não cirúrgica é contemplada (comportamental, farmacológica ou fisioterapia), o exame urodinâmico pode ser dispensável ${ }^{19}$.

O exame clínico da paciente com hipótese de bexiga hiperativa deve ser feito em posição ginecológica, preferencialmente com a bexiga cheia. A paciente deve ser solicitada a tossir e/ou realizar manobra de Valsalva. Quando há hiperatividade vesical, se houver perda, esta é assincrônica ao esforço.

A integridade dos reflexos bulbocavernoso e anocutâneo deve ser investigada, para avaliação de possivel comprometimento neurológico. Quando normais, indicam que o arco reflexo sacral $\left(\mathrm{S}_{2}\right.$, $\mathrm{S}_{3}, \mathrm{~S}_{4}$ ) e o componente motor do nervo pudendo estão preservados. Do ponto de vista prático, estes reflexos em conjunto com o tônus do esfincter anal indicam que não há comprometimento neurológico do segmento sacral. Entretanto, vale ressaltar que a falta de resposta não indica necessariamente anormalidade neurológica.

\section{Questionários de Qualidade de Vida}

Pacientes que têm perda urinária desenvolvem modificações comportamentais para se adaptar à inconveniência e reduzir o impacto dos sintomas. Incluem-se entre aqueles: aumento da freqüência urinária, descoberta da localização de banheiros, dietas restritivas, limitação da atividade física e, nos casos mais graves, limitação das atividades sociais. Isto pode resultar em isolamento secundário, não permitindo a visitação de lugares de encontro, como centros de compra, igrejas e reuniões familiares ${ }^{20}$.

Forma-se um ciclo vicioso de ansiedade e sofrimento relacionado à possivel perda urinária. Junte-se a isto o sentimento de vergonha e gradativa piora da urgência miccional pela angústia internalizada que freqüentemente leva a importante incômodo psicológico e variado grau de isolamento social ${ }^{20}$.

Instrumentos de aferição da qualidade de vida são comumente utilizados na avaliação de doenças ou de tratamentos para a determinação da percepção individual física, psicológica e bem-estar social. Em situações nas quais o objetivo principal não é o prolongamento da vida e métodos específicos de resposta ao tratamento são falhos, a medida do impacto na qualidade de vida destes pacientes é imperativa. Por esse motivo, a Sociedade Internacional de Continência recomenda que tais avaliações sejam incluídas, em todos os estudos, como complemento das medidas clínicas ${ }^{21}$.

A medida objetiva mais utilizada em estudos clínicos de incontinência urinária avalia a quantidade e freqüência da perda de urina por diários miccionais, teste do absorvente ou parâmetros urodinâmicos. Tais observações podem refletir a gravidade da perda, contudo não exprimem as alterações nas atividades diárias.

A aplicação de questionários em mulheres incontinentes é bem aceita, uma vez que o impacto sobre os fatores individuais pode ser avaliado. Para que sejam eficientes, estes instrumentos devem ser simples, ter relevância e ser de fácil compreensão. Sua interpretação é influenciada pela cultura e suas determinações deveriam ser sempre pré-testadas em população específica antes de seu uso em estudos clínicos ${ }^{22}$. De forma semelhante, a tradução e adaptação cultural devem ser enfocadas.

Dentre os questionários disponiveis, existem os que enfocam condições genéricas e específi$\operatorname{cas}^{23}$. Os questionários genéricos fornecem dados sobre o perfil individual, abrangendo conceitos de saúde geral e avaliando aspectos no campo físico, psicológico e social. Podem ser aplicados em diferentes populações, a despeito da enfermidade, tratamento proposto ou faixa etária. Tais medidas permitem comparações entre os grupos estudados, contudo não são tão sensiveis na detecção de pequenas diferenças proporcionadas pelo tratamento. The Medical Outcomes Study 36-item Short Form Health Survey (SF-36) é largamente utilizado na prática clínica, tendo sido traduzido para o português ${ }^{24}$. É multidimensional, sendo constituído por 36 itens reunidos em componentes físico e mental. Todavia, não contempla alguns aspectos como a função sexual e o sono, que são pertinentes para mulheres incontinentes.

Já os questionários específicos são desenhados para avaliar com maior complexidade e eficácia o impacto de certos aspectos clínicos e cirúrgicos. Portanto, refletem melhor a mudança na resposta ao tratamento ${ }^{22}$. 
Entre os questionários especificos, o King's Health Questionnaire (KHQ) ${ }^{25}$ é instrumento confiável e válido para aplicação em mulheres incontinentes. É constituído por oito domínios: percepção geral de saúde, impacto da incontinência urinária, limitações de atividades diárias, limitações físicas, limitações sociais, relacionamento pessoal, emoções e sono/disposição. Os valores variam de 0 a 100 e quanto maior a pontuação, pior é a qualidade de vida referente àquele critério. Temos utilizado o King's Health Questionnaire, já traduzido e validado para a lingua portuguesa, para determinação da qualidade de vida de nossas pacientes ${ }^{26}$.

Em estudo das conseqüências da bexiga hiperativa em 933 pacientes, os pesquisadores observaram que, comparada com a urgeincontinência, os sintomas da bexiga hiperativa similarmente reduziram a qualidade de vida. As pacientes com sintomas de hiperatividade vesical tiveram limitação especial com relação à sua mobilidade, enquanto a urgeincontinência relacionouse de forma mais significativa com os sentimentos de constrangimento e vergonha ${ }^{27}$.

Outros autores também avaliaram o impacto da incontinência urinária pelos questionários em estudo do tipo caso-controle com 1062 mulheres incontinentes e 1143 pacientes do grupo controle. Na avaliação da qualidade de vida, observaram significativa deterioração destes parâmetros, sendo a percepção da saúde mental e física os critérios que tiveram as mais significativas alterações à medida que os sintomas pioravam ${ }^{28}$.

Desta forma, existe crescente preocupação não somente na determinação da ocorrência de incontinência, mas também no fato de quanto este problema pode interferir no estilo de vida subjetivo de cada paciente, sendo que os questionários podem auxiliar nesta tarefa.

Na Tabela 2 estão exemplificados os principais questionários de qualidade de vida específicos para a incontinência urinária ${ }^{26,29-33}$.

Tabela 2 - Questionários específicos de qualidade de vida em mulheres com incontinência urinária (adaptado de Corcos et al. ${ }^{22}$ ).

\begin{tabular}{lccc}
\hline Questionário & $\begin{array}{c}\text { Reprodutibilidade } \\
\text { teste-reteste }\end{array}$ & Validade & $\begin{array}{c}\text { Sensibilidade } \\
\text { a mudanças }\end{array}$ \\
\hline BFLUTS & boa & boa & não relatada \\
SII & boa & moderada & não relatada \\
IIQ & moderada & boa & muito boa \\
King's Health Questionaire & boa & boa & não relatada \\
I-QOL & muito boa & boa & pouca \\
Incontinence Stress Index & não referida & moderada & não relatada \\
\hline BLUTS: Bristol Female Lower Urinary Tract Symptoms & & \\
SII: Symptom Impact Index ${ }^{20}$ & & & \\
King's Health Questionaire & & & \\
IIQ: Incontinence Impact Questionnaire & & & \\
I-QOL: Incontinence Quality of Life & & \\
ISS: Incontinence Stress Index & &
\end{tabular}

\section{Diagnóstico Urodinâmico}

O objetivo da avaliação urodinâmica é identificar as causas específicas dos sintomas das pacientes, seja o problema incontinência urinária, disfunção miccional ou sintomas irritativos do trato urinário, além de fornecer dados para orientar o correto tratamento, seja ele cirúrgico ou não. Deve-se lembrar que a avaliação urodinâmica está sujeita a artefatos, durante sua realização, que podem gerar erros de interpretação e até mesmo de diagnóstico, se o examinador não estiver atento e cuidadoso. O diagnóstico final é o resultado de contínua interação entre a paciente e o examinador, sendo de importância fundamental a interpretação dos dados e a separação de informações relativas a $\operatorname{artefatos}^{34}$.

Embora o estudo urodinâmico seja considerado o melhor método diagnóstico para avaliação da função do trato urinário, alguns autores acreditam que o mesmo não é rotineiramente necessário e preferem conduzir suas pacientes baseando-se em sintomas clínicos ${ }^{16}$. Sua utilização rotineira ainda permanece controversa. Alguns guias de conduta não o recomendam se a opção inicial de tratamento é a terapia conservadora ${ }^{35}$.

Em revisão sistemática da literatura seguida de meta-análise, foram encontrados 38 estudos potenciais para utilização do exame urodinâmico na avaliação da incontinência urinária ${ }^{36}$. Contudo, deste total apenas dois estudos foram considerados elegiveis. Na maioria se comparavam diferentes enfoques do exame, bem como havia ausência de resultados clínicos. Os autores concluíram que o número de participantes era demasiado pequeno para se afirmar se a alocação de pacientes no grupo que se submeteu ao estudo urodinâmico era significativa no resultado final após tratamento.

Para a incontinência urinária de esforço, o diagnóstico da sua classificação é essencial, uma vez que a mesma pode ser decorrente de deficiência esfincteriana ou de hipermobilidade do colo vesical. Sua diferenciação é prioritária antes de se propor tratamento cirúrgico, visto que os procedimentos de colpo-suspensão retropúbicos tradicionais podem ter indices de falha de ate $35 \%$ em pacientes com defeito esfincteriano uretral ${ }^{37}$. Não há, até o momento, padronização universal do diagnóstico ${ }^{1}$.

O termo "deficiência esfincteriana uretral intrínseca" refere-se a subtipo de IUE causada pela inabilidade do mecanismo esfincteriano uretral em manter a coaptação da mucosa tanto no repouso quanto ao esforço físico. Difere da hipermobilidade 
do colo vesical por fatores de risco diferentes, maior gravidade dos sintomas e pior resposta ao tratamento. Em geral associa-se a fatores de risco como a idade avançada, mielopatia congênita ou adquirida, hipoestrogenismo, cirurgias pélvicas radicais, radioterapia e cirurgias prévias para correção da incontinência urinária e/ou distopias genitais $^{38}$. Do ponto de vista clínico, relaciona-se com perdas aos mínimos esforços, gravitacional ou com a mudança de posição ${ }^{39,40}$.

A função esfincteriana uretral é constituída por componente ativo e outro passivo. O mecanismo passivo tem como função a manutenção da pressão de fechamento e a coaptação da mucosa em repouso. Já o mecanismo ativo mantém o tônus uretral constante e contraído durante o esforço ${ }^{41}$.

Alguns autores têm demonstrado que a pressão de perda urinária durante a manobra de Valsalva (Valsalva leak point pressure - VLPP) é método confiável para avaliação do mecanismo esfincteriano uretral. A medida da VLPP é definida como sendo a menor pressão vesical, medida com volume conhecido, na qual se observa a perda de urina, durante aumentos da pressão abdominal $^{42}$. Pacientes com IUE e VLPP inferior a 60 $\mathrm{cmH}_{2} \mathrm{O}$ provavelmente terão como etiologia a deficiência esfincteriana uretral intrínseca ${ }^{43}$. Pressões de perda superior a $90 \mathrm{cmH}_{2} \mathrm{O}$, em geral, não se relacionam com defeito esfincteriano ${ }^{43}$.

Desde a sua introdução, a determinação da pressão de perda (VLPP) tem-se mostrado clinicamente útil na avaliação do mecanismo esfincteriano e tem sido largamente utilizada como método diagnóstico ${ }^{44}$. No setor de Uroginecologia e Cirurgia Vaginal da Universidade Federal de São Paulo (UNIFESP/EPM), temos padronizado a realização do teste com volume de $200 \mathrm{~mL}$ de soro fisiológico intravesical, sendo que a paciente é solicitada a realizar, por três vezes, manobra de Valsalva gradualmente até que se observe a perda de urina, sendo medida, então, a menor pressão que causa a perda. Nos casos em que não há perda urinária descrevemos a máxima pressão atingida pela paciente. A cada $100 \mathrm{~mL}$ infundidos solicitamos a paciente tossir, observando a perda ou não de urina ao esforço. Alguns estudos mostraram que a medida da VLPP é reprodutivel desde que o método de medida e volume vesical sejam constantes, tendo sido relatada reprodutibilidade do teste em $80 \%$ de mulheres com IUE ${ }^{45}$.

Outra forma de avaliação do esfincter é feita pelo perfil pressórico uretral, obtendo-se parâmetros como a pressão máxima de fechamento uretral, comprimento funcional da uretra e pressão de transmissão abdominal à uretra. Desta forma, a pressão máxima de fechamento uretral avaliaria o mecanismo passivo. Já a pressão de perda mediria a capacidade do mecanismo ativo sob esforço ${ }^{46}$.

Avaliando 161 pacientes com o componente de esforço ao estudo urodinâmico, encontrou-se baixo coeficiente de correlação $(0,22)$ entre a medida da VLPP e da pressão máxima de fechamento uretral. Da mesma forma, observou-se fraca correlação clínica, uma vez que apenas $5 \%$ da variabilidade da pressão de perda foi dada pela variação da pressão máxima de fechamento uretral ${ }^{47}$. O baixo significado clínico pode ser atribuído ao fato de que os dois parâmetros de pressão são provavelmente avaliações de diferentes aspectos do mecanismo esfincteriano uretral.

Quanto à correlação clínica entre a gravidade dos sintomas, demonstrou-se forte associação com a medida da VLPP ${ }^{48}$. Três quartos das mulheres com sintomas importantes têm pressão de perda inferior a $90 \mathrm{cmH}_{2} \mathrm{O}$. Da mesma forma, a pressão de perda abaixo de $65 \mathrm{cmH}_{2} \mathrm{O}$ relacionouse com grau acentuado de incontinência urinária em $77 \%$ das pacientes ${ }^{49}$. Observou-se também significativa relação entre o número de pacientes com VLPP inferior a $60 \mathrm{cmH}_{2} \mathrm{O}$ e a queixa clínica, denotando a interação desta com a gravidade da incontinência. Da mesma forma, os valores médios da pressão de perda foram significativamente menores no grupo com perda aos mínimos esforços quando comparado ao grupo com perda aos grandes esforços ${ }^{50}$.

A maioria dos autores utiliza cateter eletrônico do tipo microtip para avaliação do perfil pressórico uretral, mais sensivel que os cateteres de fluxo, embora seu uso seja limitado pelos custos e pelos artefatos. Valores da pressão uretral inferiores a $20 \mathrm{cmH}_{2} \mathrm{O}$ têm sido associados a alto risco de falhas (18-54\%) em cirurgias de colpofixação retropúbica ${ }^{51}$.

Usando dados já publicados, o valor preditivo positivo da pressão máxima de fechamento uretral na predição do sucesso de procedimentos de colpofixação retropúbica variou entre $82-93 \%$, enquanto o valor preditivo negativo foi baixo, variando entre $4-53 \%{ }^{52}$.

No setor de Uroginecologia e Cirurgia Vaginal da UNIFESP/EPM, até bem pouco tempo, utilizávamos o perfil uretral com cateter de fluxo, sendo que valores inferiores a $45 \mathrm{cmH}_{2} \mathrm{O}$ na pressão máxima de fechamento uretral eram sugestivos de provável defeito esfincteriano. Contudo, não observamos interação entre o número de pacientes com valores da pressão máxima de fechamento uretral inferiores a $45 \mathrm{cmH}_{2} \mathrm{O}$ e a queixa clínica. Da mesma forma, não houve correlação entre os valores médios da pressão máxima de fechamento uretral nos diferentes grupos de mulheres 
com incontinência urinária e a queixa de perda aos esforços ${ }^{50}$. Com base nestes dados, não temos mais utilizado, de forma rotineira, o perfil uretral na prática diária.

\section{Outros Testes}

O exame de urina, tanto pela análise do sedimento quanto a urocultura, é considerado obrigatório em todas as pacientes com queixas uroginecológicas. Tal exame se faz necessário, uma vez que infecções urinárias podem ser causa dos sintomas e, com grande probabilidade, haverá manipulação instrumental do trato urinário.

O teste do absorvente ou padtest é forma objetiva de avaliar a incontinência urinária, sendo particularmente útil na documentação e quantificação da perda urinária, além de ser auxiliar na monitorização dos efeitos do tratamento ${ }^{53}$. É especialmente recomendado nos casos cuja perda não foi observada ao exame clínico nem no estudo urodinâmico. Consiste na colocação de absorvente, com seu peso previamente aferido, junto ao meato uretral externo por determinado período de tempo, durante o qual a paciente executa atividades diárias normais ou exercícios que as simulem. Após isso, o absorvente é retirado e novamente pesado. Diferenças maiores que $1 \mathrm{~g}$ caracterizam a perda urinária ${ }^{54}$.

A ultra-sonografia, em uroginecologia, tem como finalidade estimar o resíduo miccional, detectar tumores vesicais e distorções na parede vesical por massas pélvicas, avaliação da vascularização dos tecidos periuretrais por meio do efeito Doppler e da musculatura do assoalho pélvico, bem como avaliar a hipermobilidade e a posição do colo vesical $^{55-57}$. Portanto, não faz diagnóstico da incontinência urinária seja por esforço seja por hiperatividade vesical. Considera-se hipermobilidade quando há deslocamento do colo vesical acima de 1 $\mathrm{cm}$ quando comparado no repouso e ao esforço ${ }^{58}$.

\section{Conclusões}

A avaliação inadequada e o diagnóstico incorreto da etiologia da incontinência urinária têm múltiplas conseqüências, sendo as mais sérias cirurgias inapropriadas ou mesmo desnecessárias. Igualmente estressante para a paciente é a demora pelo uso de medicamentos que não surtem os efeitos desejados. Procedimentos cirúrgicos sucessivos são vistos como tendo menores índices de sucesso, além de maior risco cirúrgico e de complicações pós-operatórias.
Outro fator importante, nos dias atuais, são os custos financeiros e humanos, além das implicações médico-legais de procedimento cirúrgico desnecessário ou mesmo incorreto. Diante de quadro de hiperatividade vesical no pós-operatório, não há como se definir se esta hiperatividade já existia antes do procedimento cirúrgico ou surgiu após o mesmo, se não foi realizada a avaliação urodinâmica prévia. Ainda mais grave, se havia indicação cirúrgica para o caso e se a mesma foi a mais adequada.

O objetivo da avaliação uroginecológica é identificar as causas específicas dos sintomas das pacientes. O diagnóstico final é o resultado da contínua interação entre a paciente e o examinador, sendo de importância fundamental a interpretação dos dados e a separação de informações relativas aos artefatos.

Baseando-se nestes dados, acreditamos que a história clínica associada ao exame físico tem grande importância no manejo da incontinência urinária. Porém, não devem ser utilizados como único método diagnóstico, principalmente quando se tem em mente a possibilidade de tratamento cirúrgico. Seu valor recai sobre o rastreamento na identificação de pacientes que requerem investigação posterior. Os testes objetivos estão disponiveis e devem ser utilizados em conjunto com os dados clínicos, permitindo um diagnóstico preciso e terapia apropriada.

\section{Referências}

1. Abrams P, Cardozo L, Fall M, Griffiths D, Rosier P, Ulmsten $U$, et al. The standardisation of terminology of lower urinary tract function: report from the Standardisation Sub-committee of the International Continence Society. Am J Obstet Gynecol. 2002;187(1):116-26.

2. Fine PM, Antonini TG, Appell RA. Clinical evaluation of women with lower urinary tract dysfunction. Clin Obstet Gynecol. 2004;47(1):44-52.

3. Julian TM. Physical examination and pretreatment testing of incontinent woman. Clin Obstet Gynecol. 1998;41(3):663-71.

4. Farrar DJ, Whiteside CG, Osborne JL, TurnerWarwick RT. A urodynamic analysis of micturition symptoms in the female. Surg Gynecol Obstet. 1975;141(6):875-81.

5. Gilleran JP, Zimmern P. An evidence-based approach to the evaluation and management of stress incontinence in women. Curr Opin Urol. 2005;15(4):236-43. 
6. Heesakkers JP, Vriesema JL. The role of urodynamics in the treatment of lower urinary tract symptoms in women. Curr Opin Urol. 2005;15(4):215-21.

7. Jensen JK, Nielsen FR Jr, Ostergard DR. The role of patient history in the diagnosis of urinary incontinence. Obstet Gynecol. 1994;83(5 Pt 2):90410.

8. Haeusler G, Hanzal E, Joura E, Sam C, Koelbl H. Differential diagnosis of detrusor instability and stress-incontinence by patient history: the Gaudenz-Incontinence-Questionnaire revisited. Acta Obstet Gynecol Scand. 1995;74(8):635-7.

9. Montz FJ, Stanton SL. Q-Tip test in female urinary incontinence. Obstet Gynecol. 1986;67(2):258-60.

10. Colli E, Artibani W, Goka J, Parazzini F, Wein AJ. Are urodynamic tests useful for initial conservative management of non-neurogenic urinary incontinence? A review of the literature. Eur Urol. 2003;43(1):63-9.

11. Haylen BT, Sutherst JR, Frazer MI. Is the investigation of most stress incontinence really necessary? Br J Urol. 1989;64(2):147-9.

12. Feldner Júnior PC, Bezerra LRPS, Girão MJBC, Castro RA, Sartori MGF, Baracat ED, et al. Valor da queixa clínica e exame físico no diagnóstico da incontinência urinária. Rev Bras Ginecol Obstet. 2002;24(2):87-91.

13. Garnett S, Abrams P. The natural history of the overactive bladder and detrusor overactivity. A review of the evidence regarding the long-term outcome of the overactive bladder. J Urol. 2003;169(3):843-8.

14. Keilman LJ. Urinary incontinence: basic evaluation and management in the primary care office. Prim Care. 2005;32(3):699-722.

15. Dmochowski RR, Sanders SW, Appell RA, Nitti VW, Davila GW. Bladder-health diaries: an assessment of 3-day vs 7-day entries. BJU Int. 2005;96(7):104954.

16. Videla FL, Wall LL. Stress incontinence diagnosed without multichannel urodynamic studies. Obstet Gynecol. 1998;91(6):965-8.

17. Carey MP, Dwyer PL, Glenning PP. The sign of stress incontinence - should we believe what we see? Aust N Z J Obstet Gynaecol. 1997;37(4):436-9.

18. Weidner AC, Myers ER, Visco AG, Cundiff GW, Bump $\mathrm{RC}$. Which women with stress incontinence require urodynamic evaluation? Am J Obstet Gynecol. 2001;184(2):20-7.

19. Harvey MA, Versy E. Predictive value of clinical evaluation of stress urinary incontinence: a summary of published literature. Int Urogynecol J Pelvic Floor Dysfunct. 2001;12(1):31-7.

20. Davila GW, Neimark M. The overactive bladder: prevalence and effects on quality of life. Clin Obstet Gynecol. 2002;45(1):173-81.
21. Blaivas JG, Appell RA, Fantl JA, Leach G, McGuire EJ, Resnick NM, et al.. Standards of efficacy for evaluation of treatment outcomes in urinary incontinence: recommendations of the Urodynamic Society. Neurourol Urodyn. 1997;16(3):145-7.

22. Corcos J, Beaulieu S, Donovan J, Naughton M, Gotoh M; Symptom Quality of Life Assessment Committee of the First International Consultation on Incontinence. Quality of life assessment in men and women with urinary incontinence. J Urol. 2002;168(3):896-905.

23. Symonds T. A review of condition-specific instruments to assess the impact of urinary incontinence on health related quality of life. Eur Urol. 2003;43(3):219-25.

24. Ciconelli RM, Ferraz MB, Santos W, Meinão I, Quaresma MR. Tradução para a língua portuguesa e validação do questionário genérico de avaliação de qualidade de vida SF-36 (Brasil SF-36). Rev Bras Reumatol. 1999;39(3):143-50.

25. Kelleher CJ, Cardozo LD, Khullar V, Salvatore S. A new questionnaire to assess the quality of life of urinary incontinent women. Br J Obstet Gynaecol. 1997;104(12):1374-9.

26. Fonseca ESM, Camargo ALM, Castro RA, Sartori MGF, Fonseca MCM, Lima GR, et al. Validação do questionário de qualidade de vida (King's Health Questionnaire) em mulheres brasileiras com incontinência urinária. Rev Bras Ginecol Obstet. 2005;27(5):235-42.

27. van der Vaart $\mathrm{CH}$, de Leeuw JR, Roovers JP, Heintz AP. The effect of urinary incontinence and overactive bladder symptoms on quality of life in young women. BJU Int. 2002;90(6):544-9.

28. Chiaffarino F, Parazzini F, Lavezzari M, Giambanco V; Grupo Interdisciplinare di Studio Incontinenza Urinaria (GISIU). Impact of urinary incontinence and overactive bladder on quality of life. Eur Urol. 2003;43(5):535-8.

29. Jackson S, Donovan J, Brookes S, Eckford S, Swithinbank L, Abrams P. The Bristol Female Lower Urinary Tract Symptoms Questionnaire: development and psychometric testing. Br J Urol. 1996;77(6):80512.

30.Black N, Griffiths J, Pope C. Development of a symptom severity index and a symptom impact index for stress incontinence in women. Neurourol Urodyn. 1996;15(6):630-40.

31. Wyman JF, Elswick RK Jr, Ory MG, Wilson MS, Fantl JA. Influence of functional, urological, and environmental characteristics on urinary incontinence in community-dwelling older women. Nurs Res. 1993;42(5):270-5.

32. Wagner TH, Patrick DL, Bavendam TG, Martin ML, Buesching DP. Quality of life of persons with urinary incontinence: development of a new measure. Urology. 1996;47(1):67-72. 
33. Yu L. Incontinence stress index: measuring psychological impact. J Gerontol Nurs. 1987;13(7):1825.

34. Homma Y. The clinical significance of the urodynamic investigation in incontinence. BJU Int. 2002;90(5):489-97.

35. Fantl JA, Newman DK, Colling J, DeLancey JOL, Keeys C, Loughery $\mathrm{R}$, et al. Urinary incontinence in adults: acute and chronic management. Rockville: US Department of Health and Human Services/Public Health Service/Agency for Health Care Policy and Research; 1996. (Clinical Practice Guideline, 2; AHCPR Publ. 96-0682).

36. Glazener CM, Lapitan MC. Urodynamic investigations for management of urinary incontinence in adults. Cochrane Database Syst Rev. 2005;(3):CD003195.

37. Raz S, Sussman EM, Erickson DB, Bregg KJ, Nitti VW. The Raz bladder neck suspension: results in 206 patients. J Urol. 1992;148(3):845-50.

38. Betson LH, Siddiqui G, Bhatia NN. Intrinsic urethral sphincteric deficiency: critical analysis of various diagnostic modalities. Curr Opin Obstet Gynecol. 2003;15(5):411-7.

39. Krissi H, Pansky M, Halperin R, Langer R. Maximal urethral closure pressure < $20 \mathrm{~cm} \mathrm{H2O}$ : does it predict intrinsic sphincteric deficiency? J Reprod Med. 2005;50(11):824-6.

40.Pajoncini C, Costantini E, Guercini F, Bini V, Porena M. Clinical and urodynamic features of intrinsic sphincter deficiency. Neurourol Urodyn. 2003;22(4):264-8.

41. Ostergard DR, Bent AE. Urogynecology and urodynamics: theory and practice. 4th ed. Baltimore: Williams \& Wilkins; 1996.

42. Petrou SP, Wan J. VLPP in the evaluation of female with stress urinary incontinence. Int Urogynecol J Pelvic Floor Dysfunct. 1999;10(4):254-9.

43. McGuire EJ, Fitzpatrick CC, Wan J, Bloom D, Sanvordenker J, Ritchey M, et al. Clinical assessment of urethral sphincter function. J Urol. 1993;150(5 Pt 1):1452-4.

44. Weber AM. Leak point measurement and stress urinary incontinence. Curr Womens Health Rep. 2001;1(1):45-52.

45. Bump RC, Elser DM, Theofrastous JP, McClish DK. Valsalva leak point pressure in women with genuine stress incontinence: reproducibility, effect of catheter caliber, and correlations with other measures of urethral resistence. Continence Program for Women Research Group. Am J Obstet Gynecol. 1995;173(2):551-7.

46. Pajoncini C, Costantini E, Guercini F, Porena M. Intrinsic sphincter deficiency: do the maximum urethral closure pressure and the Valsalva leakpoint pressure identify different pathogenic mechanisms? Int Urogynecol J Pelvic Floor Dysfunct. 2002;13(1):30-5.
47. Feldner PC Jr, Bezerra LR, de Castro RA, Sartori MG, Baracat EC, de Lima GR, et al. Correlation between valsalva leak point pressure and maximal urethral closure pressure in women with stress urinary incontinence. Int Urogynecol J Pelvic Floor Dysfunct. 2004;15(3):194-7.

48. Bai SW, Chung KA, Rha KH, Kim SU, Kim SK, Park $\mathrm{KH}$. Correlation between urodynamic test results, perineal ultrasound and degree of stress urinary incontinence. J Reprod Med. 2003;48(9):718-22

49. Cummings JM, Boullier JA, Parra RO, WozniakPetrofsky J. Leak point pressures in women with urinary stress incontinence: correlation with patient history. J Urol. 1997;157(3):818-20.

50. Feldner Júnior PC, Bezerra LRPS, Girão MJBC, Castro RA, Sartori MGF, Baracat EC et al. Correlação entre a pressão de perda à manobra de Valsalva e a pressão máxima de fechamento uretral com a história clínica em mulheres com incontinência urinária de esforço. Rev Bras Ginecol Obstet. 2002;24(7):433-8.

51.Lemack GE. Urodynamic assessment of patients with stress incontinence: how effective are urethral pressure profilometry and abdominal leak point pressures at case selection and predicting outcome? Curr Opin Urol. 2004;14(6):307-11.

52. Weber AM. Is urethral pressure profilometry a useful diagnostic test for stress urinary incontinence? Obstet Gynecol Surv. 2001;56(11):720-35.

53. Paick JS, Ku JH, Shin JW, Park K, Son H, Oh SJ, et al. Significance of pad test loss for the evaluation of women with urinary incontinence. Neurourol Urodyn. 2005;24(1):39-43.

54. Soroka D, Drutz HP, Glazener CM, Hay-Smith EJ, Ross S. Perineal pad test in evaluating outcome of treatments for female incontinence: a systematic review. Int Urogynecol J Pelvic Floor Dysfunct. 2002;13(3):165-75.

55. Girão MJBC, Takano CC, Castro RA, et al. Incontinência urinária de esforço. In: Baracat EC, Rodrigues de Lima R, editores. Guias de medicina ambulatorial e hospitalar - UNIFESP/ Escola Paulista de Medicina. São Paulo: Manole; 2005. p.189-99.

56. Jarmy-Di Bella ZI, Girão MJ, Sartori MF, Di Bella Junior V, Lederman HM, Baracat EC, et al. Power Doppler of the urethra in continent or incontinent, pre- and postmenopausal women. Int Urogynecol J Pelvic Floor Dysfunct. 2000;11(3):148-54.

57. Girão MJ, Jarmy-Di Bella ZI, Sartori MG, Baracat EC, Lima GR. Doppler velocimetry parameters of periurethral vessels in postmenopausal incontinent women receiving estrogen replacement. Int Urogynecol J Pelvic Floor Dysfunct. 2001;12(4):241-6.

58. Girão MJBC, Sartori MGF, Baracat EC, Rodrigues de Lima G. Incontinência urinária de esforço. In: Rodrigues de Lima R, Girão MJBC, Baracat EC, editores. Ginecologia de consultório. São Paulo: EPM/Editora de Projetos Médicos; 2003. p. 27-35. 